\section{Gold in Polyaniline: Recent Trends}

\author{
Kaushik Mallick, ${ }^{1}$ Mike J Witcomb, ${ }^{2}$ \\ Mike S Scurrell ${ }^{1 *}$ \\ ${ }^{1}$ Molecular Sciences Institute, School of Chemistry, \\ 2 Electron Microscope Unit, \\ University of the Witwatersrand Johannesburg, Private \\ Bag 3, WITS 2050, South Africa. \\ ** scurrell@aurum.wits.ac.za
}

\begin{abstract}
Nanotechnology offers an extremely broad range of potential applications in, for example, electronics, optical communication and biological systems. Gold nanoparticles of different sizes and shapes combined with polyaniline can give rise to a host polymer with interesting physical properties and important potential applications. Many possible applications for this system have been explored, many devices and systems have been studied, while more potential applications and devices are being proposed. Such composites are discussed in this review on the basis of the techniques used for their synthesis, their properties and possible applications.
\end{abstract}

\section{Introduction}

The discovery of conductive polymers has opened up many new possibilities for devices which combine unique optical, electrical, and mechanical properties. Existing knowledge (14) relating to conducting polymers reveals the diversity of materials that can be used, optical effects achieved, and underlying physical processes. Conducting polymers include organic polymers, co-polymers, and conjugated polymers. Examples of the conducting polymers are poly ( $p$-phenylene), polyaniline, poly ( $p$-phenylenevinylene) etc. These polymers can be fabricated to have a high degree of flexibility. The devices using them are mainly light-emitting diodes (LEDs) and lasers for which the color of the light emitted can be chemically tuned. The main physical process involved is electroluminescence.

Among conducting plastics, polyaniline is of high interest due to its outstanding properties. It is one of the so-called doped polymers in which conductivity results from a process of partial oxidation or reduction. Polyaniline compounds can be designed to achieve the required conductivity for a given application. The resultant blends can be as conductive as silicon and germanium or as insulating as glass. Another advantage is that the compound can be simply mixed with conventional polymers and it is easy to fabricate polyaniline products into specific shapes. The conductivity of polyaniline makes it an ideal shield against static electricity discharges and thus polyaniline compounds have been used in the packaging of electronics products. Polyaniline compounds are being tested for use as protection against electromagnetic radiation. Further, scientists hope that one day printed circuit boards, electrochromic windows in houses and cars, and conductive fabrics will contain polyaniline compounds. It is well known that polyaniline compounds provide protection against corrosion.

Polyaniline has become a focal point of interest because of its environmental stability (5), controllable electrical conductivity (6), and interesting redox properties associated with the chain nitrogen (7). The electrical properties of the aniline polymers can be substantially improved through secondary doping (8). Their excellent processability, together with the presence of a number of intrinsic redox states (Figure 1), have substantially enhanced the potential applications of aniline polymers for use in practical devices. Polyaniline is a member of the semi-flexible rod polymer family. The synthesis and characterization of electroactive polymers have become two of the most important areas of research in polymers as well as in materials science over the past two decades.

The aniline polymers have a general formula of the type $\left[(-\mathrm{B}-\mathrm{NH}-\mathrm{B}-\mathrm{NH}-)_{\mathrm{y}}(-\mathrm{B}-\mathrm{N}=\mathrm{Q}=\mathrm{N}-)_{1-\mathrm{y}}\right]_{x}$, in which $\mathrm{B}$ and $\mathrm{Q}$ denote respectively the $\mathrm{C}_{6} \mathrm{H}_{4}$ rings in the benzenoid and the quinonoid forms. In polyaniline, the neutral intrinsic redox states (Figure 1) can vary from that of the fully oxidized pernigraniline $(y=0)$, to that of the fully reduced leucoemeraldine $(y=1)$. The $50 \%$ intrinsically oxidized 


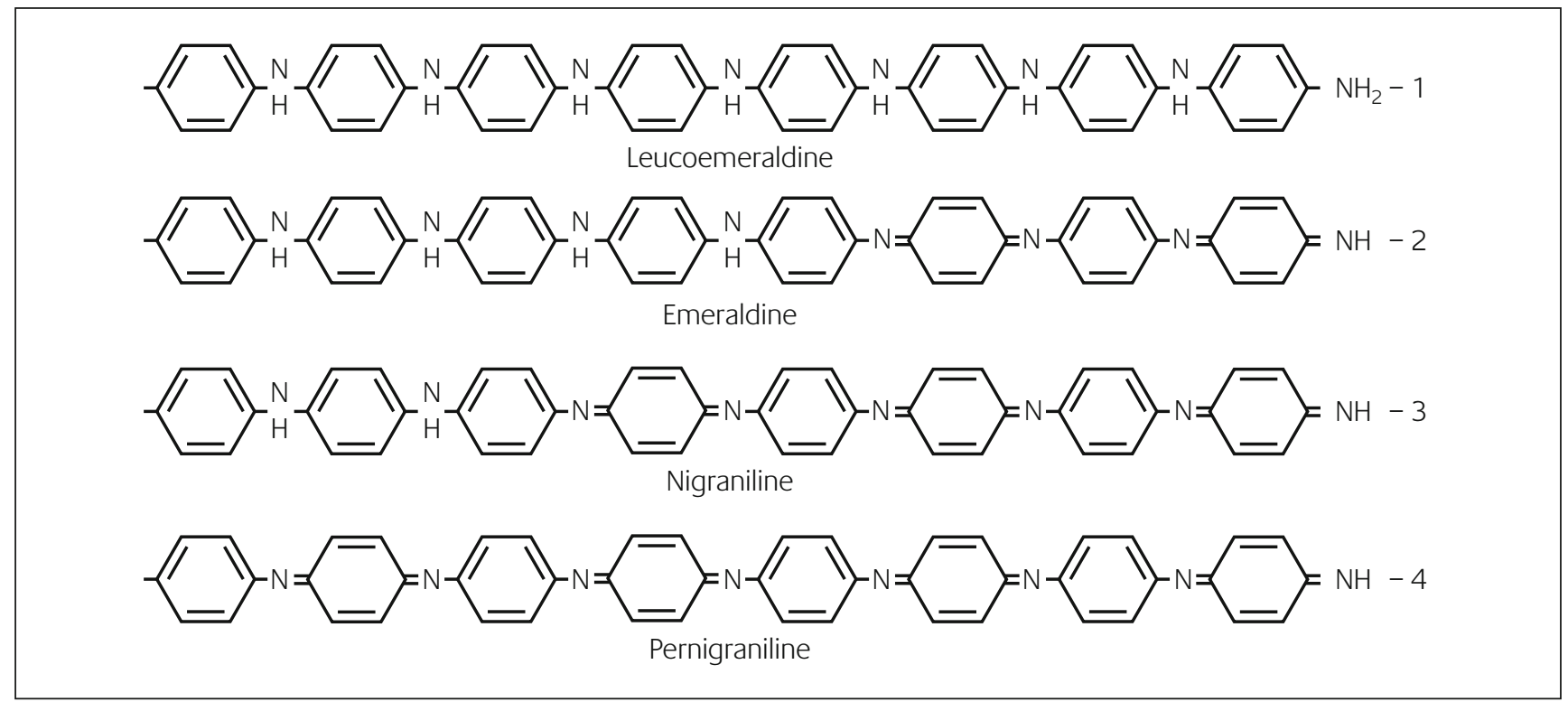

Figure 1

The various intrinsic oxidation states of polyaniline (from ref 9)

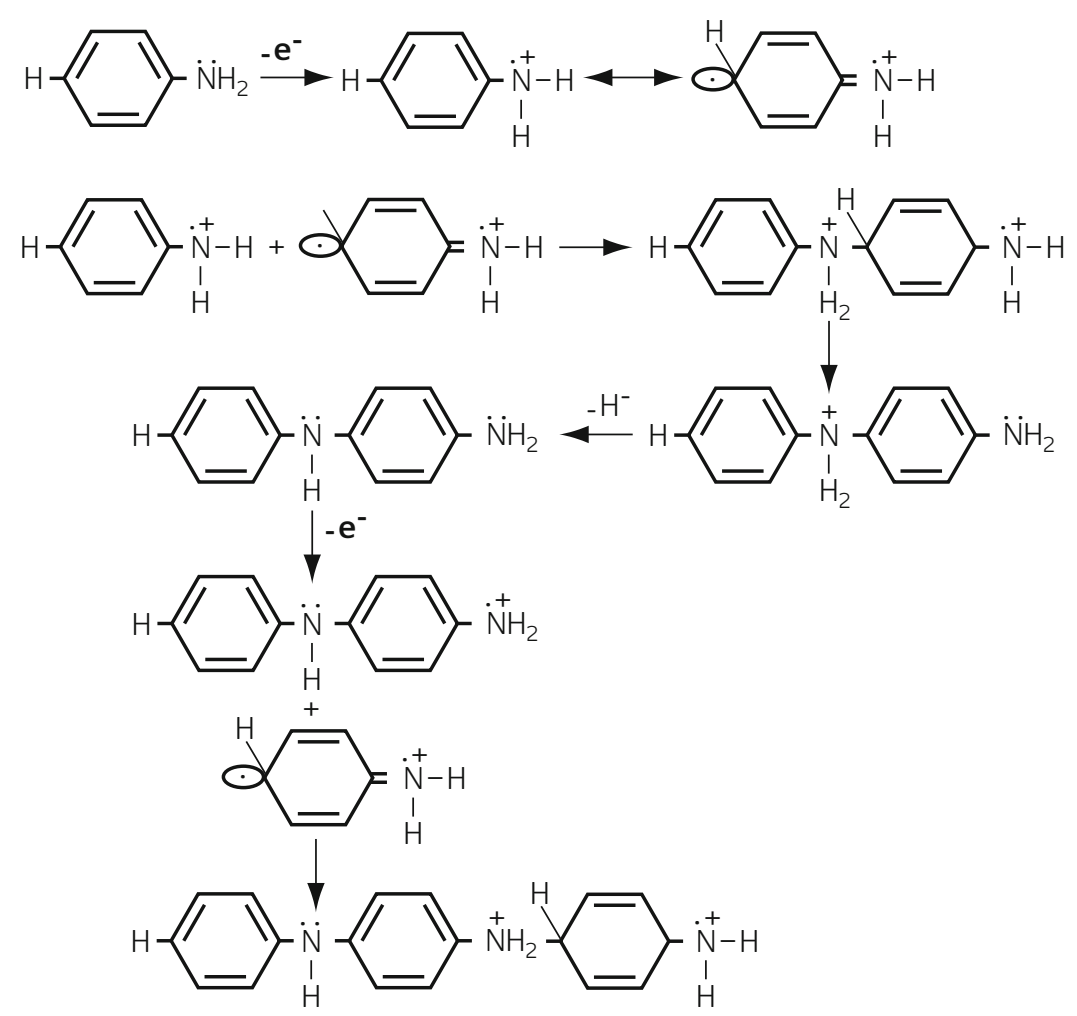

\section{Figure 2}

Mechanistic pathway for the polymerization of aniline

polymer has been termed emeraldine ( $y=0.5)$, and the $75 \%$ intrinsically oxidized polymer, is termed nigraniline $(y=0.75)$ (9). Figure 2 shows the mechanism involved in the polymerization of aniline. The synthesis and characterization of polyaniline have been reviewed by Genies et al. (10), Lux (11) and Gospodinova et al. (12). These reviews dealt with chemical, electrochemical and gas-phase preparations, polymerization mechanisms, physiochemical and electrochemical properties, redox mechanisms, theoretical studies as well as applications of the polymer.
Advances in materials science have taken a new direction since the advent of nanoparticle-based materials. Nanoparticles have properties that are different from those of their atomic/molecular and bulk counterparts. Nanocomposites are a special class of materials originating from suitable combinations of two or more such nanoparticles or nanosize objects by some suitable technique, which results in materials having unique physiochemical properties and a large potential for applications in diverse areas. Novel properties of 
nanocomposites can be derived from the successful combination of the characteristics of the parent constituents into a single material. To exploit nanomaterials for technological applications it is very important to endow them with good processability through using a suitable matrix. Conventional polymers usually serve as the matrix, which results in a special class of hybrid materials termed "polymeric nanocomposites".

The present review will restrict its field of reference solely to gold-polyaniline composite materials. The major breakthrough in the synthesis methods coupled with their application related performance in various systems will be discussed.

\section{Preparation of the gold-polyaniline composite material}

Incorporation of gold nanoparticles into polyaniline has become a popular and interesting aspect of composite synthesis and a number of different methods have been suggested. This material differs from the pure polyaniline in respect of some of the physical and chemical properties. The synthesis of gold-polyaniline composite materials was pioneered by Kang and co-workers (13). They recorded the spontaneous and sustained reduction and accumulation of elemental gold from a chloroauric acid solution by electroactive polyaniline in the leucoemeraldine and emeraldine oxidation states. They observed that the reduction rate was substantially retarded by the loss of active surface area as a result of the gold coverage. Eight years later, Kang's group (14) published a more comprehensive and precise work for the synthesis of a gold nanoparticle and polyaniline composite material in which they reported on two sets of experiments involving the emeraldine form of polyaniline in both $\mathrm{N}$-methylpyrrolidinone and in an aqueous medium. $\mathrm{AuCl}_{3}$ was used as the precursor of the gold nanoparticles. Maintaining the molar ratio of (metal ion) : ( $N$ of emeraldine) at 1:1, 1:5, 1:10 and 1:20, they concluded, through employing laser light scattering and electron microscopy, that the largest particle size was obtained when the metal ion: $\mathrm{N}$ was close to $1: 5$. When the metal salt reduction was carried out in a $\mathrm{N}$-methylpyrrolidinone solution of polyaniline, around 20 nm diameter metal particles were obtained while the polyaniline was converted to a higher oxidative state. The reduction of metal salts using polyaniline powder in aqueous media yielded larger particles that were in the size range $50-200 \mathrm{~nm}$. In the case of both methods, the size of the metal particles was dependent on the reaction time, reaction medium, and initial metal ion to polyaniline ratio.

Chattopadhyay et al. (15) have reported on the preparation of a gold-polyaniline composite material by using hydrogen peroxide as both the oxidizing and the reducing agent. This two step process involved the reduction of $\mathrm{HAuCl}_{4}$ to Au nanoparticles in an aqueous medium followed by the polymerization of aniline which results in the formation of a composite of gold nanoparticles and conductive polyaniline.
The material was characterized by means of UV-vis spectrophotometry, FTIR, ${ }^{1} \mathrm{H}$ NMR and XRD. A Fourier Transform IR spectrum of the composite showed strong absorbance at 1490 and $1570 \mathrm{~cm}^{-1}$, which are features that are characteristic of the deformation modes of the benzenoid and quinoid rings respectively. The ${ }^{1} \mathrm{H}$ NMR spectrum of the composite material confirmed the presence of the benzenoid and quinoid forms of the emeraldine salt. The incorporation of gold nanoparticles into the polyaniline moiety was confirmed by powder X-ray diffraction. Using the Debye-Scherrer equation, the size of the nanoparticles was estimated to be $26 \mathrm{~nm}$. The electrical conductivity of the gold nanoparticle-polyaniline composite was measured to be $0.3 \mathrm{~S} \mathrm{~cm}^{-1}$.

Such nanoparticle-polymer composites are of interest for the generation of organic (16) and inorganic (17) supramolecular assemblies for encapsulation of guest molecules with intended application in solubilization (18), catalysis (19) and controlled delivery (20). Among the popular choices in this regard are three dimensional fluid structures such as micelles, vesicles, and the helical structures of starch (21). The linear analogue of starch offers a unique advantage in this respect since it is a biopolymer. The polymer can form a helical structure with a hydrophobic core that can accommodate hydrophobic moieties while the molecule itself can be dissolved in water. By exploiting this property of starch, Sarma and Chattopadhyay (22) introduced such a strategy and reported on the results of the solubilization of polyaniline in water by encapsulation in starch as well as a polyaniline-gold-nanoparticle composite. The work is an extension of their previous work described in (15). TEM studies revealed that the gold particle sizes were in the range $10-20 \mathrm{~nm}$.

Although the chemical synthesis of polyaniline using

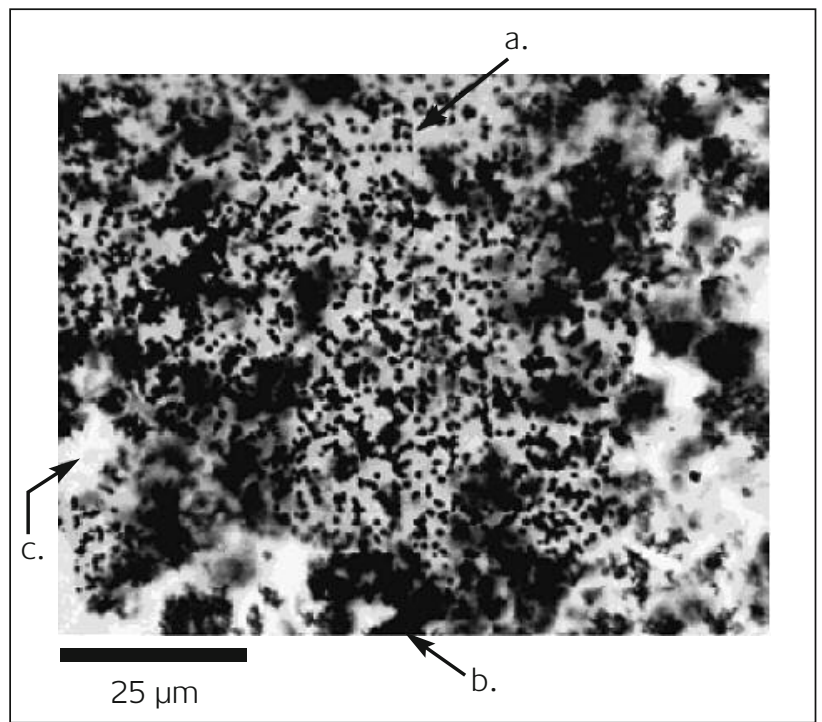

Figure 3

Transmitted light optical image of the Au/PANI composite in the reaction solution: (a) gold particles (black dots) encapsulated in the polymer (light gray regions); (b) optically dense region of the PANI/Au composite; (c) void space (absence of $A u(0)$ or PANI) - reproduced with permission from ref 26 
ammonium peroxydisulfate [23-25] is well characterized, the synthesis of polyaniline using $\mathrm{AuCl}_{4}{ }^{-}$is more complex in terms of a mechanistic point of view. Issues such as the final oxidation state of the metal and polymer, degree of proton doping, degree of incorporation of the metal into the polymer matrix, and chemical composition of the polymer have to be addressed. A detailed study of a synthetic route for producing a gold-polyaniline composite material using $\mathrm{AuCl}_{4}{ }^{-}$as the oxidizing agent in solutions with $\mathrm{HBF}_{4}$ added as a doping agent has been reported (26). The conductance of the resultant gold-polyaniline composite doped with $\mathrm{HBF}_{4}$ was $3.2 \mathrm{~S} \mathrm{~cm}^{-1}$ which compares to a conductivity of only $4.4 \times 10^{-7} \mathrm{~S} \mathrm{~cm}^{-1}$ for the undoped composite. The data suggested that proton doping is the critical step in maintaining the conductivity of gold-polyaniline composite. The data also indicated that the single-step synthesis of a highly conductive gold-polyaniline composite is possible using $\mathrm{AuCl}_{4}{ }^{-}$as the oxidant in the presence of suitable doped acid. TEM images revealed that the diameter of the gold particles was relatively constant at about $1 \mu \mathrm{m}$ (Figure 3). This synthesis route provides a method for producing gram quantities of gold-polyaniline composite material with a high metal content (up to 54 wt \%) coupled with good chemical reproducibility.

Many methods for the synthesis of metal nanoparticles have been tried. The conventional techniques employed for the synthesis of metal nanoparticles have been chemical reduction, UV-irradiation, $\gamma$-irradiation etc. Highly uniform and well-dispersed metal nanoparticles can be produced by using such procedures and $\gamma$-irradiation is one of the powerful techniques for the synthesis of well dispersed metal nanoparticles. For example, Pillalamarri et al. (27) reported the synthesis method for gold-polyaniline composite materials in which polyaniline fibers were decorated with gold nanoparticles by using such a technique. Different concentrations of $\mathrm{HAuCl}_{4}$ were used as the precursor for the gold nanoparticles. The samples were irradiated with $\boldsymbol{\gamma}$-rays, the reactor being operated at $180 \mathrm{~kW}$ while the radiation dose rates were varied from an initial value of about $1.5 \mathrm{kGyh}^{-1}$ to about $0.3 \mathrm{kGyh}^{-1}$ at the end of the irradiation period. The irradiated polyaniline fibers had diameters of 50 $100 \mathrm{~nm}$ and lengths of $1-3 \mu \mathrm{m}$. The gold particle size varied from 10-100 nm depending on the aniline/ $\mathrm{HAuCl}_{4}$ ratio. XRD patterns of the polyaniline-gold composite exhibited intense peaks corresponding to the (111), (200), (220), and (310) Bragg reflections of gold. The FT-IR spectra of the composite exhibited absorption peaks at $1570 \mathrm{~cm}^{-1}$ and $1490 \mathrm{~cm}^{-1}$ that corresponded to the stretching modes of the quinonoid and benzenoid rings respectively, as well as $\mathrm{N}-\mathrm{H}$ stretching at $3400 \mathrm{~cm}^{-1}$. The conductivity of the composite material improved with increasing metal loading. By increasing the $\mathrm{HAuCl}_{4}$ concentration from $0.002 \mathrm{M}$ to $0.02 \mathrm{M}$, the conductivity of the composite material increased from $0.1 \mathrm{~S}$ $\mathrm{cm}^{-1}$ to $1.0 \mathrm{~S} \mathrm{~cm}^{-1}$. The authors concluded that the shapes and the sizes of the metal nanoparticles could be varied by changing the metal-to-aniline ratio. The electrical conductivity of the composites was found to be up to 50 times higher than that of pure polyaniline nanofibers.

Different morphologies of polyaniline have been obtained utilizing various synthesis or processing routes. In recent years, one-dimensional (1-D) polyaniline nanostructures, including nanofibers and nanotubes have attracted considerable research interest since they are of desirable molecular wire form as well as having a controllable conductivity. One dimensional polyaniline nanostructures have been synthesized chemically or electrochemically through a template synthesis route. Different templates such as zeolite channels (28), track-etched polymer membranes (29), anodized alumina membranes $(30,31)$, or soft templates including surfactants (32) and micelles (33) have been employed to direct the growth of the 1-D nanostructures of polyaniline. Recently, some new methods have been reported for the synthesis of such structured polyaniline. For example, an interfacial polymerization method has been applied to prepare polyaniline fibers at the interface of solvents with different polarity (34). Wang et al. (35) reported an interesting finding in which $\mathrm{HAuCl}_{4}$ was used as an oxidizing agent for the synthesis of polyaniline nanofibers. A simple reaction at room temperature between an acidic aqueous solution of aniline and $\mathrm{a} \mathrm{HAuCl}_{4}$ solution resulted in the simultaneous formation of polyaniline nanofibers and gold particles. The ensuing product was characterized by spectroscopy and electron microscopy. The UV-vis spectrum (Figure 4a) of the product which was dispersed in water showed two prominent adsorption peaks at 340 and $800 \mathrm{~nm}$ with a shoulder at around $410 \mathrm{~nm}$. Although in the paper there is no explanation of the characteristic spectral behavior of the compound, it is well documented that the absorption peak at $340 \mathrm{~nm}$ is due to the $\pi-\pi^{*}$ transition of the benzenoid rings. A shoulder-like appearance at $410 \mathrm{~nm}$ is due to the polaron / bipolaron transition. The absorption peak at $800 \mathrm{~nm}$ is a consequence of the benzenoid to quinoid excitonic transition. The morphology of the resultant polyaniline was studied by

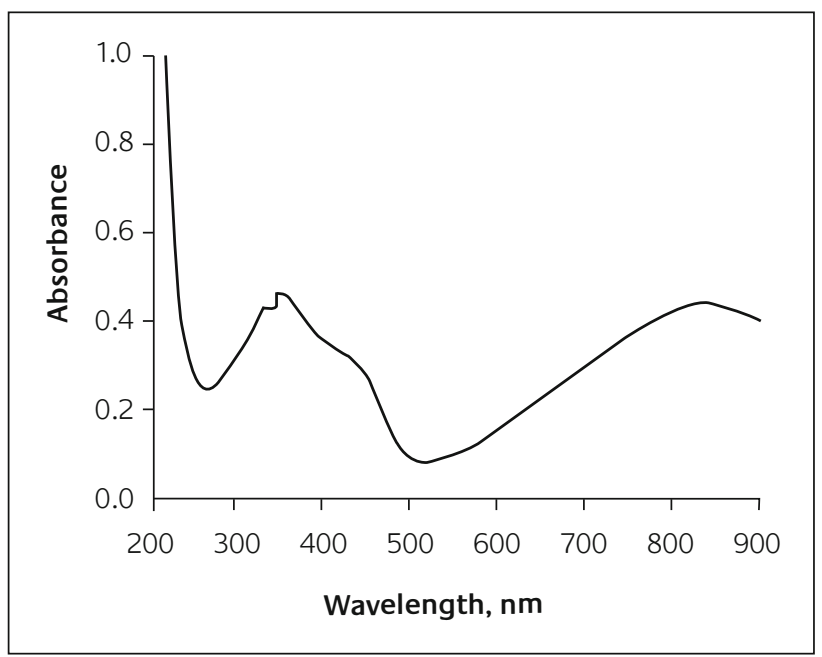

Figure 4a

UV-vis spectrum of the dark green product (PANI) in water - reproduced with permission from ref 35 

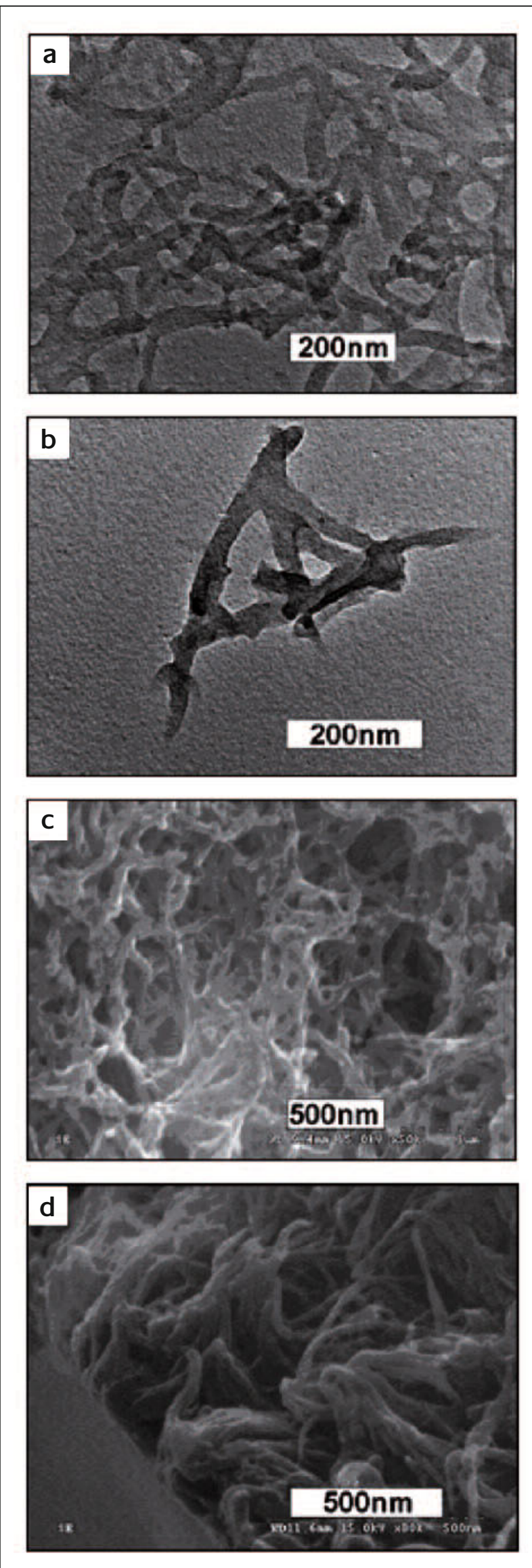

\section{Figure 4b}

(a) TEM image of PANI nanofibers. (b) Higher magnification TEM image of a branched network of PANI nanofibers. (c) SEM image of PANI nanofibers. (d) Cross-sectional view of a PANI nanofiber film - reproduced with permission from ref 35 electron microscopy. Both the TEM and SEM images (Figure $4 \mathrm{~b}$ ) indicated that the resultant polyaniline consisted of uniform nanofibers with a diameter around $35 \mathrm{~nm}$, the length of the fibers ranging from hundreds of nanometers to several micrometers. The authors concluded from transmission electron microscopy (TEM) and energy dispersive $x$-ray spectroscopy (EDX) that the gold particles were coated by a thin polymeric sheath yielding a core-shell composite material consisting of a gold core and polyaniline shell. The conductivity of the resultant composite was measured to be $\sim 0.16 \mathrm{~S} \mathrm{~cm}^{-1}$.

Methods have been found for the synthesis of polyanilinegold nanocomposites by using the 'grafting from' approach of Pillalamarri et al. (36). The preformed gold nanoparticles were stabilized using a surfactant with a peroxide group. The peroxide initiated polymerization when gold nanoparticles were added to the aniline. Two types of nanosized gold, spheres and rods were used to build the composite material. According to the authors, the oligomers were formed initially in the proximity of the gold surfaces since the peroxide initiator is attached to the gold nanoparticles. The next step involved the catalytic activity at the surfaces of the particles, the presence of the oligomers playing a role in the fiber formation. The procedure allowed control over the polyaniline and gold through an improved attachment due to the nanoscopic dimensions of both components.

Polyaniline can be produced with different types of morphologies such as fibers, hollow-spheres, tubes etc. Recently, our group has reported on the formation of polyaniline nanoballs decorated with the gold nanoparticles (37). $\mathrm{HAuCl}_{4}$ was used as the oxidizing agent and was
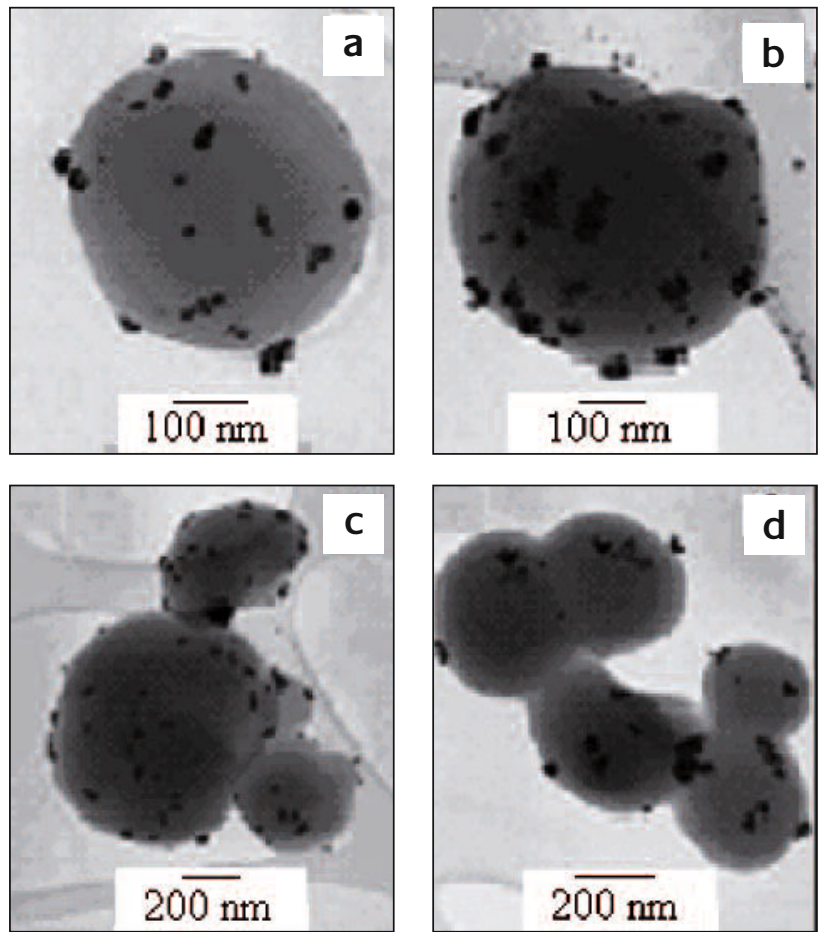

Figure 5a

TEM image of the precipitated gold-polyaniline material. The dark spots are the gold particles which decorate the polyaniline nanoballs - reproduced with permission from ref 37 


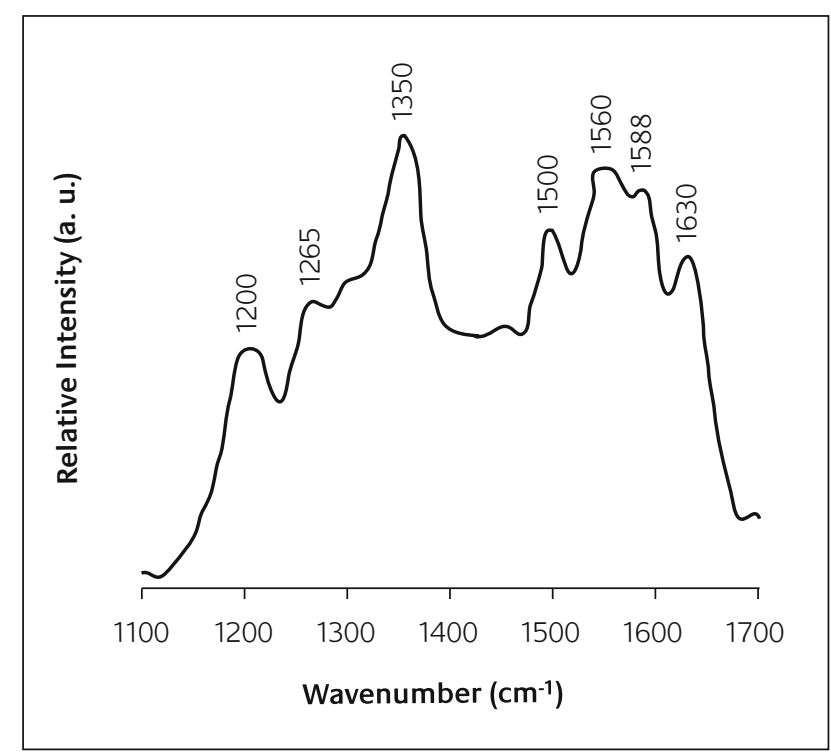

\section{Figure $5 b$}

Raman spectrum obtained from the precipitated gold particle covered polyaniline nanoball - reproduced with permission from ref 37

dissolved in toluene by using a phase transfer catalyst. A TEM image of the resulting product (Figure $5 \mathrm{a}$ ) revealed the formation of nanoballs which were decorated by the gold nanoparticles (dark spots). In the Raman spectrum (Figure 5b), the characteristic bands from 1100 to $1700 \mathrm{~cm}^{-1}$ are sensitive to the oxidation state of the polyaniline. The spectrum of the composite polymer revealed the C-C deformation bands of the benzenoid ring at 1630, 1588 and $1560 \mathrm{~cm}^{-1}$ which are characteristic of the semiquinone rings. The peak at about $1500 \mathrm{~cm}^{-1}$ corresponds to the $\mathrm{N}-\mathrm{H}$ bending deformation band of the protonated amine while the band at $1350 \mathrm{~cm}^{-1}$ corresponds to the $\mathrm{C}-\mathrm{N}^{\cdot+}$ stretching mode of the delocalized polaronic charge carriers. The band at $1265 \mathrm{~cm}^{-1}$ was assigned to the $\mathrm{C}-\mathrm{N}$ stretching mode of the polaronic units. The broad overlapping feature in the range of 1150 to $1230 \mathrm{~cm}^{-1}$ seen with a peak at $1200 \mathrm{~cm}^{-1}$ was due to the convolution of peaks at 1171 and $1221 \mathrm{~cm}^{-1}$, and attributed to the $\mathrm{C}-\mathrm{H}$ bending deformation and $\mathrm{C}-\mathrm{N}$

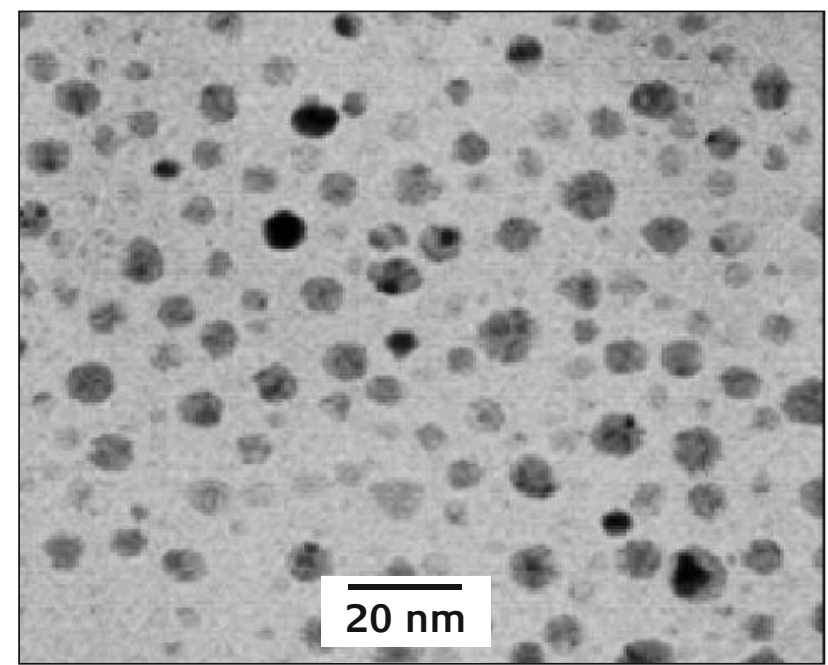

\section{Figure 6a}

TEM image of the gold-polyaniline composite material revealing highly dispersed gold nanoparticles - reproduced with permission from ref 38

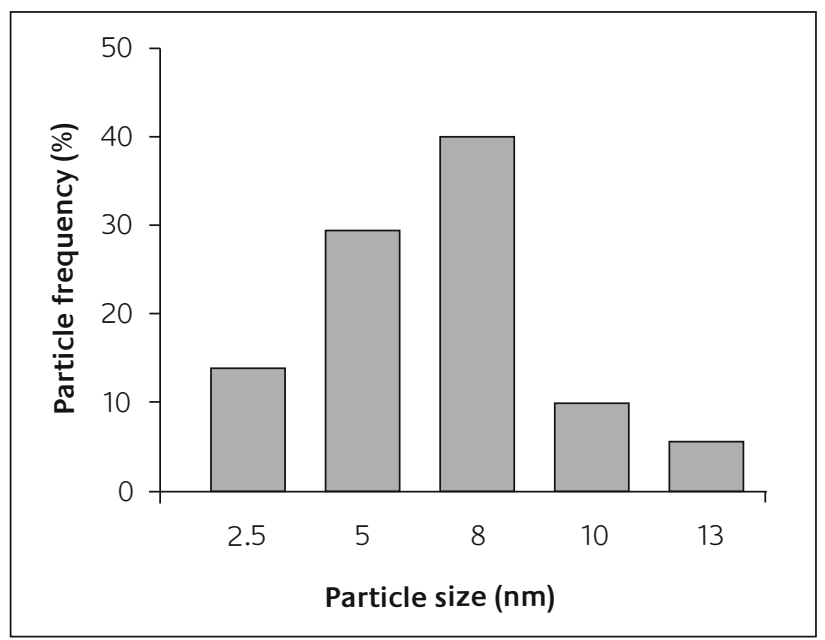

Figure $6 b$

Histogram (particle size vs. particle frequency) indicates the size distribution of the gold nanoparticles, which are present in metalpolymer composite material. The histogram is calculated on the basis of figure $6 B$ - reproduced with permission from ref 38

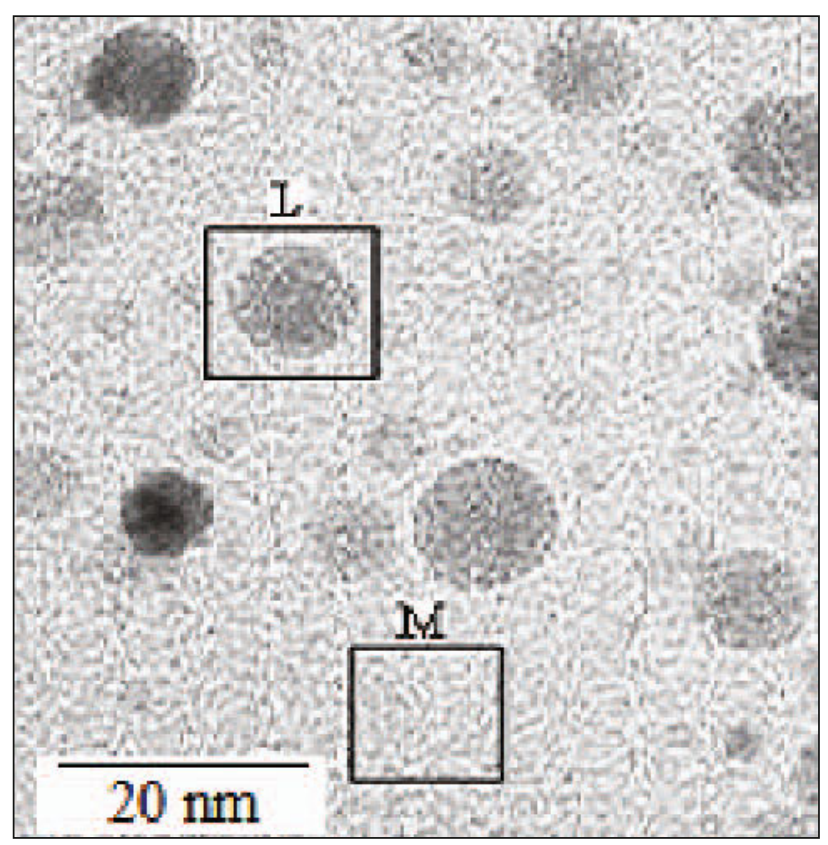

\section{Figure 6c}

High magnification TEM image of the gold-polyaniline composite - reproduced with permission from ref 38

stretching modes respectively. When $\mathrm{HAuCl}_{4}$ was dissolved in water and aniline was diluted in methanol, no such microstructured morphology of the gold-polyaniline composite material was obtained (38). Figure $6 \mathrm{a}$ is a TEM image showing the gold-polyaniline composite where it can be seen that the gold nanoparticles are highly dispersed in the polymer matrix. The histogram in Figure $6 \mathrm{~b}$ reveals that $80 \%$ of the gold particles are within the size range $5-10 \mathrm{~nm}$. Figure $6 \mathrm{c}$ is a magnified image of the composite material in which area $\mathrm{M}$ indicates the morphology of the polymer and a similar morphology has also been observed on the gold particles. Tilting experiments and studies of the edge of the composite reveal that most of the gold nanoparticles are covered by the polyaniline.

Polymers based on polyaniline derivatives have attracted 


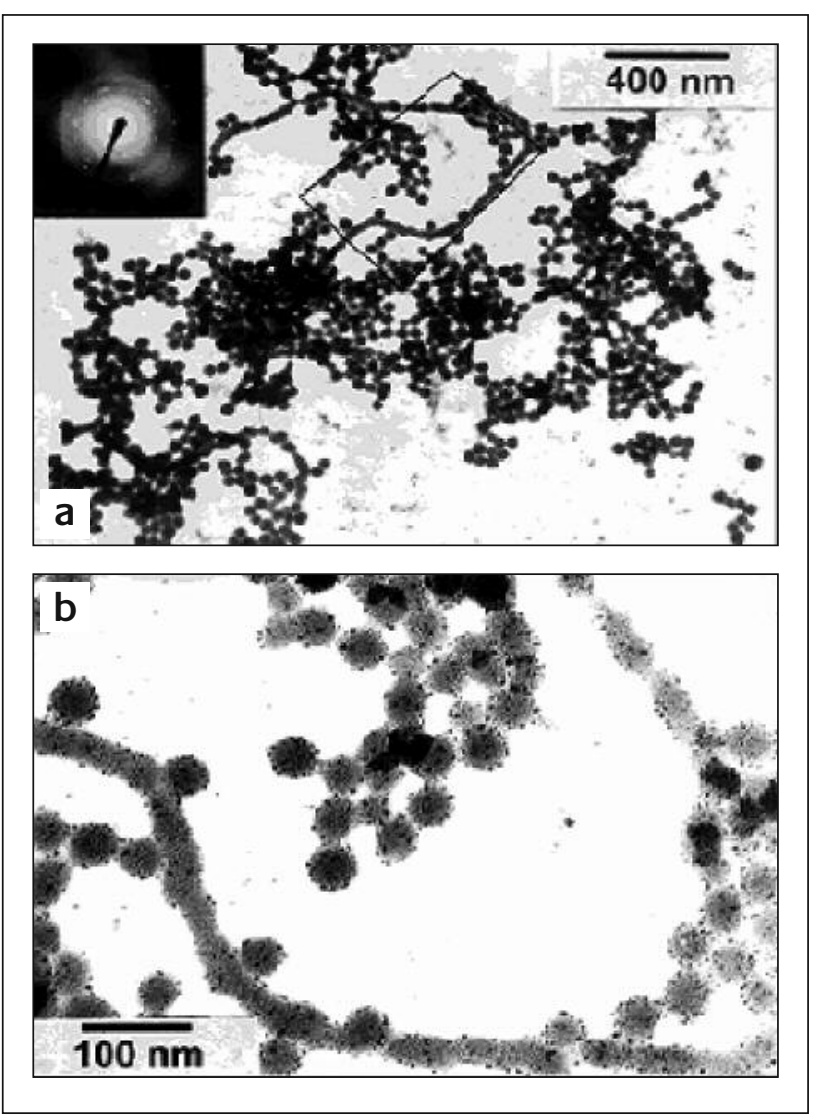

Figure 7

(a) A representative TEM image of poly(o-anisidine) micelles decorated by Au nanoparticles prepared in an aqueous solution by method I of ref 39. (b) An enlargement of a part of (a) to reveal the Au nanoparticles wrapped in the poly(o-anisidine) micelles. The inset in (a) is a selected area diffraction pattern from the gold nanoparticles in a poly(o-anisidine) micelle - reproduced with permission from ref 39

growing scientific attention because their chemical properties are similar to those of polyaniline and they are more easily processed than the parent polymer.

There have been reports of derivatives of aniline being polymerized using $\mathrm{HAuCl}_{4}$ to form gold-polymer composite materials which underwent a similar type of synthetic mechanism. For example, Dai et al. (39) synthesized a gold-poly(o-anisidine) composite using $o$-anisidine as the reducing agent. The main aim of that work was to synthesize gold nanoparticles by using o-anisidine, but during the synthetic process a composite of gold-poly(o-anisidine) was produced. Emphasis was focused in that paper on the structure and properties of the gold nanoparticles, but no effort has been made to investigate the morphology of the gold-poly(o-anisidine) composite material (Figure 7). It was concluded that formation of poly(o-anisidine) was in the form of the emeraldine salt (the conducting state), this being supported by UV-vis spectroscopy, IR spectroscopy and XPS.

Sun et al. (40) have reported a simple route for the large scale and rapid synthesis of uniform poly(o-phenylenediamine) nanobelts by mixing $\mathrm{HAuCl}_{4}$ and o-phenylenediamine aqueous solutions at room temperature without the additional introduction of templates. The authors suggested that the spontaneous formation of the nanobelts was due to gold nanoparticles-catalyzed oriented growth of poly(o-phenylenediamine). The reduction of $\mathrm{HAuCl}_{4}$ by o-phenylenediamine leads to the formation of gold nanoparticles and o-phenylenediamine oligomers. Subsequently, the gold nanoparticles served as catalysts active in the oriented oxidative polymerization of other o-phenylenediamine monomers by $\mathrm{HAuCl}_{4}$ along the oligomers. This reaction resulted in the formation of poly(o-phenylenediamine) nanobelts of several hundred micrometers in length as well as isolated gold nanoparticles.

\section{Applications for gold-polyaniline composite materials}

Applications of composite materials are based on the physical properties of the host as well as the guest material. The size effect of both components of the composite material offers additional possibilities for manipulation and provides room for accommodating multiple functionalities. This section provides some examples that have been explored to illustrate the wide range of possible applications of gold-polyaniline composite materials.

\section{Electrochemical oxidation of ascorbic acid and glucose oxidase}

Granot et al. (41) reported on a detailed study of using a gold-polyaniline hybrid system for the electrochemical oxidation of ascorbic acid and glucose oxidase. The composite material consisted of sulfonate-functionalized gold nanoparticles incorporated into polyaniline and its application was for enhanced electrocatalysis. Two types of gold-polyaniline composites were generated, one configuration being a planar film, while the other was rodshaped. It was found that the electrocatalyzed oxidation of ascorbic acid by the gold-polyaniline composite was some 200\% more efficient than for the polyaniline alone. For the electrocatalytic oxidation of glucose, the gold-polyaniline system is close to five times more active than the polyaniline without gold. The improved catalytic activity was attributed to the enhanced charge transport through the gold nanoparticles. The system was further developed by microstructuring of the gold-polyaniline composite. The electrocatalytic oxidation of glucose by the micro-rod shaped gold-polyaniline composite had a 10-fold higher catalytic activity when compared to that of the polyaniline. This electrocatalytic oxidation enhancement was attributed to the higher surface area of the microstructured polymers. This increase in surface area of the electroactive polymers which was exposed to the electrolyte solution facilitated the electrical contacting of the solubilized reactant species, resulting in the improved electrocatalysis.

\section{Oxidation of $\beta$-nicotinamide adenine dinucleotide (NADH)}

A new procedure for the synthesis of a gold-polyaniline composite has been reported by Tian et al. (42a) in which 
polyaniline was doped with mercaptosuccinic-acid-capped gold nanoparticles using a layer-by-layer deposition method The gold-polyaniline multilayer films revealed an excellent redox activity and a good electrocatalytic efficiency with regard to the oxidation of $\beta$-nicotinamide adenine dinucleotide (NADH).

\section{Detection of DNA hybridization}

Another advantage of doping polyaniline with mercaptosuccinic-acid-capped gold nanoparticles is that the carboxyl groups on the mercaptosuccinic-acid can be used to link enzymes or other biomolecules to the composite film. Tian et al. (42b) demonstrated this for such a treated polyaniline film when it was utilized for the detection of DNA hybridization. Firstly covalent attaching of the aminoterminated DNA catcher probes ( $\mathrm{NH}_{2}$-DNA) to the carboxyl groups of the mercaptosuccinic-acid was undertaken followed by the monitoring of their hybridization with different DNA target strands using both electrochemical methods and surface plasmon enhanced fluorescence spectroscopy.

\section{Electrocatalytic oxidation of Dopamine}

A composite of hollow sphere polyaniline and gold nanoparticles is an interesting material from a synthesis point of view. Recently, Feng et al. (43) reported the synthesis route for such a composite material and found an important application for it in the detection of dopamine (DA), an important neurotransmitter in mammalian central nervous systems. Polystyrene (PS) particles were prepared from a styrene monomer by using potassium persulphate. Polystyrene was sulphonated using concentrated sulfuric acid to form wet PS-sulfonated PS core-shell gel particles. An aniline monomer was added to the mixture. The addition of aqueous ammonium persulphate resulted in the formation of polyaniline coated PS core/shell particles. The polyaniline hollow spheres were obtained by dissolving PS cores in THF. The gold colloid was prepared using trisodium citrate. Polyaniline hollow spheres were added to the gold colloid and adsorbed on the surface of polyaniline, leading to the formation of gold-polyaniline composite hollow spheres. The conductivity of the pure polyaniline hollow spheres with a shell thickness of $30 \mathrm{~nm}$ was $2.25 \times 10^{-3} \mathrm{Scm}^{-1}$ whereas the conductivity of gold-polyaniline composite hollow spheres with a shell thickness of $30 \mathrm{~nm}$ was about four times higher at $8.74 \times 10^{-3} \mathrm{~S} \mathrm{~cm}^{-1}$. When the gold nanoparticle content was increased from 5.7 to $10.3 \%$, the conductivity of the composite increased to $9.83 \times 10^{-3} \mathrm{~S} \mathrm{~cm}^{-1}$ clearly indicating the beneficial effect of adding gold to improve the conductivity of polyaniline. In the electrocatalytic oxidation of DA, it was found that the catalytic effect of the goldpolyaniline composite increased with the number of gold nanoparticles. Further, when the size of gold nanoparticles was less than $30 \mathrm{~nm}$, the catalytic effect was found to increase, but it decreased drastically when the size of gold nanoparticles was larger than $30 \mathrm{~nm}$. The improved electrocatalytic oxidation of DA in the gold-polyaniline modified electrode was due to the enhanced charge transport through the gold-polyaniline composite system. The enhanced electron transfer was attributed to the charge hopping through the metallic conductor gold nanoparticles that mediated the effective charge migration through the polymer. The gold nanoparticles generated many active sites for charge transfer through the interface inside the electrode by making good contact with the polyaniline matrix.

\section{Non-volatile memory chips}

Conjugated polymers and other organic materials are uniquely suited for low cost electronic devices particularly in organic light-emitting diodes (44), transistors (45), solar cells (46) and memory devices (47). By using nanoscale materials, high density electronic devices are possible with superior performance and manufacturability (48). A conducting polymer decorated with metallic or semiconducting nanoparticles provides an exciting system with which to study the possibility of designing device functionality.

Tseng and co-workers (49) fabricated a polymer based memory element using polyaniline nanofibers decorated with gold nanoparticles. A relatively uniform distribution of gold nanoparticles was created by controlling the time and the temperature of the reaction between $30 \mathrm{~nm}$ diameter polyaniline nanofibers and chloroauric acid. The device was reported to show a promising behavior, a prolonged retention time of several days with respect to applications in digital nonvolatile memory chips. The operational mechanism most likely involves an electric-field-induced charge transfer between the polyaniline nanofibers and the gold nanoparticles. The authors projected that such a polymer based memory device could have an important impact in the near future for information technology by providing high speed, high density memories essential for advanced computers and digital electronics.

\section{Conclusions}

Gold-polyaniline composite materials are a very important addition to the list of novel materials with unique functionality. From the standpoint of synthesis techniques, its properties and its wide scope of application, this composite material has raised a great deal of scientific and technological interest and promises to lead a new hugely versatile direction of research in materials. By varying the morphology of the component and the synthetic partner, the properties of the composite material can be improved beyond that possible by simple mixing and blending with molecular mixing being obviously capable of yielding more interesting structures. Considering the progress of research in this field, it can be concluded that, in the near future, gold-polyaniline composites will produce further significant advances in the field of materials science. 


\section{Acknowledgements}

K.M. expresses thanks to the University of the Witwatersrand and the National Research Foundation for the award of postdoctoral fellowships.

\section{References}

1 D. Braun, A.J. Heeger, Appl. Phys. Lett., 1991, 58, 1982

2 G. Gustafsson, Y. Cao, G.M. Treacy, F. Klavetter, N. Colaneri, A.J. Heeger, Nature, 1992, 35, 477

3 N.C. Greenham, S.C. Moratti, D.D.C. Bradley, R.H. Friend, A.B. Holmes, Nature, 1993, 365, 628

4 G. Grem, G. Leditzky, B. Ullrich, G. Leising, Adv. Mater., 1992, 4, 36

5 K. Amano, H. Ishikawa, A. Kobayashi, M. Satoh, E. Hasegawa, Synth. Met., 1994, 62, 229

6 A. Ray, G.E. Asturias, D.L. Kershner, A.F. Richter, A.G. MacDiarmid, A.J. Epstein, Synth. Met., 1989, 29, E141

7 A.H-L. Geoff, M.C. Bernar, Synth. Met., 1993, 60, 115

8 A.G. MacDiarmid, A.J. Epstein, Synth. Met., 1994, 65, 103

9 E.T. Kang, K.G. Neoh, K.L. Tan, Prog. Polym. Sci., 1998, 23, 277

10 E.M. Genies, A. Boyle, M. Lapkowski, C. Tsintavis, Synth. Met., 1990, 36, 139

11 F. Lux, Polymer, 1994, 35, 2915

12 N. Gospodinova, L. Terlemezyan, Prog. Polym. Sci., 1998, 23, 1443

13 E.T. Kang, Y.P. Ting, K.G. Neoh, K.L. Tan, Polymer, 1993, 34, 4994

14 J. Wang, K.G. Neoh, E.T. Kang, J. Colloid Interface Sci., 2001, 239, 78

15 T.K. Sarma, D. Chowdhury, A. Paul, A. Chattopadhyay, Chem. Commun., 2002, 1048

16 G. Li, W. Fudickar, M. Skupin, A. Klyszcz, C. Draeger, M. Lauer, J.H. Fuhrhop, Angew. Chem., Int. Ed., 2002, 41, 1828

17 R. Gangopadhyay, A. De, Chem. Mater. 200, 12, 608 (and references therein)

$18 \mathrm{~J}-\mathrm{H}$. Fuhrhop, J. Koning, in Monographs in Supramolecular Chemistry; Molecular Assemblies and Membranes: The Synkinetic Approach; J.F. Stoddart, Ed., Royal Society of Chemistry: London, 1994

19 S.W. Huang, K.G. Neoh, E.T. Kang, H.S. Han, K.L. Tan, J. Mat. Chem. 1998, 8, 1743

20 M. Kramer, J.F. Stumbe, H. Turk, S. Krause, A. Komp, L. Delineau, S. Prokhorova, H. Kautz, R. Haag, Angew. Chem. Int. Ed., 2002, 41, 4252
21 D. Balasubramanian, B. Raman, B.C.V. Sundari, J. Am. Chem. Soc., 1993, 115, 74

22 T.K. Sarma, A. Chattopadhyay, Langmuir, 2004, 20, 4733

23 M. Angelopoulos, G.E. Asturias, S.P. Ermer, A. Ray, E.M. Scherr, A.G. Macdiarmid, M. Akhtar, Z. Kiss, A.J. Epstein, Mol. Cryst. Liq. Cryst., 1988, 160, 151

24 S. Quillard, G. Louarn, S. Lefrant, A.G. Macdiarmid, Phys. Rev. B., 1994, 50,12496

25 A.A. Syed, M. K. Dinesan, Talanta, 1991, 38, 815

26 J.M. Kinyanjui, D.W. Hatchett, J.A. Smith, M. Josowicz, Chem. Mater., 2004, 16, 3390

27 S.K. Pillalamarri, F.D. Blum, A.T. Tokuhiro, M.F. Bertino, Chem. Mater., 2005, 17, 941

28 C.G. Wu, T. Bein, Science, 1994, 264, 1757

30 C.R. Martin, Chem. Mater., 1996, 8, 1739

31 C.W. Wang, Z. Wang, M.K. Li, H.L. Li, Chem. Phys. Lett., 2001, 341, 431

29 R.V. Rarthasarathy, C.R. Martin, Chem. Mater., 1994, 6, 1627

32 J.C. Michaelson, A.J. McEvoy, Chem. Commun.,1994, 79

33 H.J. Qiu, M.X. Wan, J. Polym. Sci., Part A: Polym. Chem., 2001, 39, 3485

34 J.X. Huang, S. Virji, B.H. Weiller, R.B. Kaner, J. Am. Chem. Soc., 2003, 125,314

35 Y. Wang, Z. Liu, B. Han, Z. Sun, Y. Huang, G. Yang, Langmuir, 2005, 21, 833

36 S.K. Pillalamarri, F.D. Blum, M.F. Bertino, Chem. Commun., 2005, 4584

37 K. Mallick, M.J. Witcomb, A. Dinsmore, M.S. Scurrell, Macromol. Rapid Commun., 2005, 26, 232.

38 K. Mallick, M.J. Witcomb, M.S. Scurrell, J. Mater. Sci. 2006, 41, 6189

39 X. Dai, Y. Tan, J. Xu, Langmuir, 2002, 18, 9010

40 X. Sun, S. Dong, E. Wang, Chem. Commun., 2004, 1182

41 E. Granot, E. Katz, B. Basnar, I. Willner, Chem. Mater., 2005, 17, 4600

42 (a) S. Tian, J. Liu, T. Zhu, W. Knoll, Chem. Commun., 2003, 2738 (b) S. Tian, J. Liu, T. Zhu, W. Knoll, Chem. Mater., 2004, 16, 4103

43 X. Feng, C. Mao, G. Yang, W. Hou, J-J. Zhu, Langmuir, 2006, 22, 4384

44 R.H. Friend, R.W. Gymer, A.B. Holmes, J.H. Burroughes, R.N. Marks, C. Taliani, D D.C. Bradley, D A. Dos Santos, J.L. Bre' das, M. Lögdlund, W.R. Salaneck, Nature, 1999, 397, 121

45 H. Sirringhuas, N. Tessler, R.H. Friend, Science, 1998, 280, 1741

46 G. Yu, J. Gao, J.C. Hummelen, F. Wudl, A.J. Heeger, Science, 1995, 270, 1789

47 J.C. Scott, Science, 2004, 304, 62

48 A.P. Alivisatos, Science, 1996, 271, 933

49 R.J. Tseng, J. Huang, J. Ouyang, R.B. Kaner, Y. Yang, Nano Lett., 2005, 5, 1077 